\title{
Extraction of Aspect-Independent Parameters Using Spectrogram Method for Chipless Frequency-Coded RFID
}

\author{
Zeshan Ali, Etienne Perret, Senior Member, IEEE, Nicolas Barbot, and Romain Siragusa
}

\begin{abstract}
In this paper, a novel method for the extraction of aspectindependent parameters (that are analogous to complex natural resonances) of chipless radio frequency (RF) identification (RFID) tags is presented. This method is based on short-time Fourier transform (STFT). The concept is proved by utilizing classical depolarizing tags. Owing to the proposed spectrogram approach, the reading success rate of chipless RFID tag is improved by accurate extraction of identification (ID) based on the extraction of quality factors. With single tag measurement (i.e., without empty measurement) in a practical environment, the performance of the proposed spectrogram method is characterized: 1) at various misalignments of the reader and the tag. 2) at various distances for the tag mounted on numerous objects. 3) at various displacements within 3D zone of reader interrogation. The proposed technique is computationally fast due to the inherent nature of fast Fourier transform (FFT) based STFT.
\end{abstract}

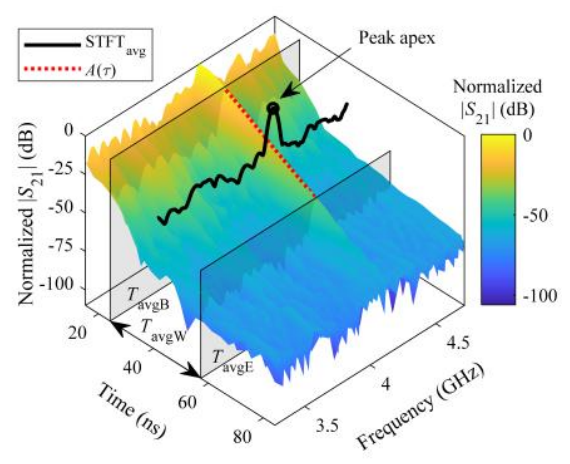

Index Terms - Chipless radio frequency (RF) identification (RFID), matrix pencil method (MPM), quality factor, short-time Fourier transform (STFT), singularity expansion method (SEM).

\section{Introduction}

$\mathrm{C}$ HIPLESS radiofrequency (RF) identification (RFID) technology has emerged as a potential tool for item-level tagging [1]. In addition to the identification, the chipless RFID technology is also being utilized for wireless sensing for environmental monitoring and industrial control. These sensing applications are, for example, humidity sensing [2][5], temperature and $\mathrm{CO}_{2}$ sensing [6], temperature sensing [7], [8], fluid level detection [9], permittivity sensing of different materials [10]-[12] (e.g., civil structural health), strain and crack sensing [13], [14], submillimeter displacement sensing [15], gesture recognition [16], 2D localization sensing [17][19], touch event sensing [20], and coating defect detection and corrosion prediction [21]. Many of these sensing capabilities are achieved by quantizing the minor shifts of the peak apexes associated with scatterers present in the design of chipless RFID tags. So, the requirement of robust detection techniques has appeared as one of the significant challenges

This work was supported in part by the Univ. Grenoble Alpes via the AGIR program, the Région Auvergne-Rhône-Alpes via the ARC6 program, and the Institut Universitaire de France. This project has also received funding from the European Research Council (ERC) under the European Union's Horizon 2020 Research and Innovation Program (grant agreement No 772539, SCATTERERID).

Zeshan Ali, Nicolas Barbot, and Romain Siragusa, are with the Univ. Grenoble Alpes, Grenoble INP, LCIS, 26000 Valence, France (e-mail: zeshan.ali@lcis.grenoble-inp.fr).

Etienne Perret is with the Univ. Grenoble Alpes, Grenoble INP, LCIS, 26000 Valence, France and also with the Institut Universitaire de France, 75005 Paris, France (e-mail: etienne.perret@lcis.grenobleinp.fr). for the practical implementation of chipless RFID technology. The robust detection techniques are needed because the chipless tag's response is generally very low compared to the backscattering response from surrounding unknown objects. Also, the growing interest of increasing the coding capacity of chipless RFID tags (see [22, Table III]) implies the requirement of the robust detection techniques. The reason behind this statement is that in frequency-coded chipless RFID, the high coding capacity involves the increase in the number of peak apexes (associated with resonant scatterers) in the limited ultrawideband (UWB). This congestion in the limited UWB would consequently narrow the allocated band to each peak apex that serves as a coding channel for each scatterer. With such narrow and closely positioned coding channels operating in a practical environment, the peak apexes might be shifted from their required position due to the superposition of reflections comings from the presence of unknown objects surrounding the chipless tag. These uncontrolled shifts in the position of peak apexes might lead to a decoding error for high density chipless tags. With less robust detection techniques, the increase in the coding capacity might not be beneficial for the operation in a practical environment. Furthermore, the tag identification (ID) reading in chipless RFID technology is much challenging as compared to in the ultra-high frequency (UHF) RFID technology, because of the absence of time modulation scheme.

To date, most of the scientific work done in the chipless RFID field is based on two measurements: tag measurement and empty measurement (i.e., in the absence of chipless tag). Background normalization is postprocedure on the measured 
signals. This postprocedure is based on the subtraction of the empty measurement from the tag measurement. In practical cases with rapidly varying background objects, the background normalization might be very difficult to do. In these conditions, merely the systematic signal distortion can be removed: the signals coming from static objects and the antenna coupling. Apart from this, a slight change in the aspect angle (human error) or the size of to-be-tagged object might also produce random shifts in the peak apexes from their nominal position and the spurious peak apexes. Strictly speaking, in practical cases, the peak apexes do not correspond to the frequencies of resonances of scatterers. Without robust detection techniques, the extracted values are not independent of the measurement setup and vulnerable to decoding error. For the rest of this paper, the signals with background normalization (i.e., subtraction of the empty measurement from the tag measurement) and the signals without background normalization are referred to as the calibrated signals and the uncalibrated signals, respectively.

This paper proposes a simple approach to overcome the above-discussed challenges. The basic idea is linked to the fact that the backscattered field from the tag's scatterer originates late in time after the coupling and structural mode (i.e., direct optical reflections). It will be shown that this late time response is linked to complex natural resonances (CNRs) or poles of the scatterers. Furthermore, the singularity expansion method (SEM) [23] states that CNRs are aspectindependent information (i.e., not dependent on the excitation or aspect). Such an aspect-independent nature of the pole(s) of a scatterer body (i.e., late time response) can be analyzed by SEM in the complex frequency plane (S-plane). The positions of poles in S-plane are independent. So, the late time response of chipless tag will be used in this paper to extract the tag ID in a rigorous way: first extracting the aspect-independent poles (where each pole contains both the frequency of resonance and quality factor) and then deciding the accurate frequency of resonance in each coding channel based on the corresponding quality factor. This procedure is done in order to obtain better accuracy compatible with the reading of high density chipless RFID tags.

Table I summarizes the state of the art of detection techniques for chipless RFID tags. In the literature, the matrix pencil method (MPM) [24] is one of the methods to extract CNRs of a scatterer to implement SEM. For the characterization of tag's ID in chipless RFID technology using CNRs, MPM [25] and its variant short-time matrix pencil method (STMPM) [26] have already been discussed. In [27], STMPM is applied to extract the high-dense tag code. In [28], the early-time and late-time modes of the transient response from multi-scatterer targets have been distinguished using STMPM. In [29], the tag code of the mobile chipless RFID tags is extracted using the inverse synthetic aperture radar (ISAR) data processing. The chipless tags are also attached to the plastic box filled in a controlled way with tape rolls. On the other hand, the mother wavelet is applied to extract the ID of tag mounted on various objects [30], where the tag motion at different stepped positions is also considered. It is important to note that all these works [25]-[30] are based on the calibrated signals (i.e., with background normalization).

Next, the uncalibrated signals (i.e., without background normalization) based techniques are discussed. A time and frequency domains (TD-FD) analysis [31] and a scalar method [32] are presented without attaching the tag on objects and without considering aspect angles. Two works in the literature [33], [34] are based on the short-time Fourier transform (STFT). The tag is attached to the metallic object and also held in the hand in [33]. The tag is attached to wooden slab and the reader-tag polarization and rotation angle are studied in [34]. In [35], a mathematical model is presented to decode the tag ID, where the tag is mounted on a plastic jig placed in front of a metallic plate.

In this paper, a novel method for the extraction of aspectindependent parameters (that are analogous to CNRs) is presented. The proposed method is based on STFT, which is referred to as the spectrogram method. The concept is proved by using depolarizing chipless RFID tags. A summary of previous work on the depolarizing chipless RFID tags and STFT is as follows: 1) in [36], the depolarizing chipless RFID tags have been proposed to suppress the effect of clutter from the reflective background objects. The tag ID is extracted using two-measurements. 2) A technique based on STFT has been proposed for the chipless RFID tag detection. With this technique, the tag ID is extracted using a single tagmeasurement (i.e., without background normalization). However, the error range of the extracted peak apexes due to the different aspect angles (human error) and the irregular tobe-labeled objects has not been discussed in [33]. Therefore, the focus of this paper is to show that the method presented in [33] can be extended to extract aspect-independent parameters such as frequency of resonance and damping factor. However, these parameters extracted by the proposed spectrogram method (extended STFT averaging method) reflect the same information as CNRs. For the first time, we bring the proof that this method can be used to obtain CNRs. The extraction of quality factor $Q$ is not discussed in [33]. Although $Q$ (i.e., alternatively damping factor) is not used in information coding, it is extremely beneficial in the decoding of the tag ID as introduced in this paper. The spurious peak apexes can be ignored using the extracted $Q$. Hence, $Q$ is useful in the decoding of tag ID. This concept is introduced for the first time in this paper.

TABLE I

StATE OF THE ART OF DETECTION TECHNIQUES FOR CHIPLESS RFID TAGS.

\begin{tabular}{|c|c|c|c|}
\hline Techniques & Signals & Objects & Aspect angles \\
\hline MPM [25] & Calibrated & No & No \\
\hline STMPM [26]-[28] & Calibrated & No & No \\
\hline ISAR processing [29] & Calibrated & $\begin{array}{l}\text { Plastic box filled } \\
\text { with tape rolls }\end{array}$ & $\begin{array}{l}\text { Tag motion on a } \\
\text { conveyer belt }\end{array}$ \\
\hline Mother wavelet [30] & Calibrated & Various & $\begin{array}{l}\text { Tag motion at } \\
\text { different stepped } \\
\text { positions }\end{array}$ \\
\hline TD-FD analysis [31] & Uncalibrated & No & No \\
\hline Scalar method [32] & Uncalibrated & No & No \\
\hline STFT [33] & Uncalibrated $_{t}^{\mathrm{I}}$ & $\begin{array}{l}\text { Metallic object, and } \\
\text { tag held in the hand }\end{array}$ & No \\
\hline STFT [34] & Uncalibrated & Wooden slab & $\begin{array}{l}\text { Reader-tag } \\
\text { polarization and } \\
\text { rotation angle }\end{array}$ \\
\hline Mathematical model [35] & Uncalibrated & $\begin{array}{l}\text { Plastic jig in front } \\
\text { of a metallic plate }\end{array}$ & No \\
\hline $\begin{array}{l}\text { Spectrogram method } \\
\text { (this work) }\end{array}$ & Uncalibrated & $\begin{array}{l}\text { Various including } \\
\text { worst cases }\end{array}$ & $\begin{array}{c}\text { Various including } \\
\text { worst cases }\end{array}$ \\
\hline
\end{tabular}


For the first time, we present the tag ID detection and decoding method using uncalibrated signals with tag mounted on various objects presenting various aspect angles. In this work, the tag is directly attached to a water tank (highly lossy) and a metallic plate (highly reflective), in contrast to [35], where the tag is not directly mounted on the metallic plate. Also, we will see that compared to the proposed spectrogram method, MPM (or STMPM) is not so simple to implement for single tag measurement. Also, MPM is a time-consuming approach that is not compatible with real-time reading.

The organization of this paper is as follows. Section II explains the employed chipless tags along with the measurement setup in a realistic room environment. Also, this Section discusses the tag reading challenges and the extraction of a priori parameters. Section III introduces the algorithm of the proposed spectrogram method. For comparison with the proposed method, a brief introduction of MPM is also presented. Section IV presents an analytical description of various kinds of superpositions between the tag mode and the structural mode. Next, this Section presents the performance of the proposed method to extract the tag code independent from the orientation of the reader (or orientation of the tag). The minimum detectability limit of the distance between the tag and antenna and the tag azimuth angle is characterized. Subsequently, the tag code independent from various to-belabeled objects and the tag code for the signals measured on 3D measurement bench are presented. Section V draws conclusions.

\section{Chipless RFID Tags, Measurement Setup, AND TAG ID DECODING CHALLENGES}

In this work, the employed chipless RFID tag is the depolarizing RF Elementary Particle (REP) tag. This tag is based on shorted dipoles oriented at $45^{\circ}$ presented in [36]. The layout of this tag is depicted in Fig. 1(a). The mutual coupling among such scatterers is discussed in [37], where two identical resonators are coupled along $x$ with distance $r_{1}$ and along $y$ with distance $r_{2}$ as shown in Fig. 1(b) and (c), respectively. For the resonators coupled along $x$, with $r_{1}$ ranging from $15 \mathrm{~mm}$ to $40 \mathrm{~mm}$, the mean value of peak apex of electric field equals to $0.43 \mathrm{~V} / \mathrm{m}$ with a variability of around $0.1 \mathrm{~V} / \mathrm{m}$. On the other hand, the magnitude of peak apex of the electric field varies from 0.49 to $0.57 \mathrm{~V} / \mathrm{m}(1.1 \mathrm{~dB})$, while $r_{2}$ varies from $25 \mathrm{~mm}$ to $40 \mathrm{~mm}$ for the resonators coupled along $y$. Furthermore, the scatterers [see Fig. 1(a)] are designed and optimized for the radar cross section (RCS). Each scatterer is realized from multiple coupled dipoles. The same resonance characteristics can also be achieved from a patch scatterer exhibiting a size equal to the overall size of the multiple coupled dipoles scatterer. One main reason for coupling the multiple dipoles is to suppress the spurious resonance peak which happens due to the currents propagating in the direction $x$ in a microstrip patch [see local $x y$ coordinates system in Fig. 1(a)]. A detailed discussion on the selection criteria of the number of dipoles to make a scatterer can be seen in [37, Chap. 4].

The measurement setup used in a realistic room environment is shown in Fig. 2. For the experimental results, an Agilent N5222A vector network analyzer (VNA) with an output power of $-5 \mathrm{dBm}$ is used as a reader. The frequency sweep ranging from 2 to $8 \mathrm{GHz}$ with 10001 points is used. An open boundary quad-ridge dual-polarization antenna (Satimo QH2000) is connected to VNA on ports 1 and 2, in vertical and horizontal polarization, respectively. The isolation between ports of this antenna is greater than $30 \mathrm{~dB}$. The distance between the tag and the antenna is $r=10 \mathrm{~cm}$. The measured quantity is the transmission coefficient $S_{21}$. For these initial measurements, the tag is attached to foam support which does not backscatter any part of the incident signal. The antenna can rotate with reading interrogation angle $\alpha_{\mathrm{T}}$ keeping the tag fixed at its position.

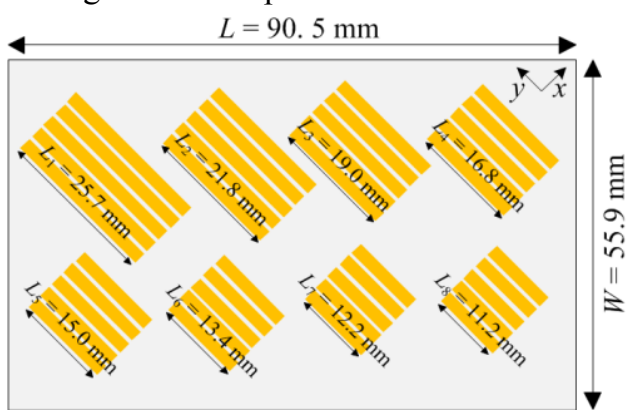

(a)

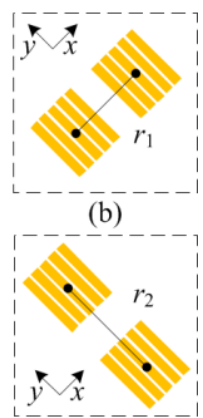

(c)
Fig. 1. (a) Layout of the employed depolarizing chipless tag. The order of scatterers is taken as ascending order from the largest scatterer to the smallest scatterer. Two similar resonators are (b) coupled along $x$ and (c) coupled along $y$. A local $x y$ coordinates system is attached to the resonators.
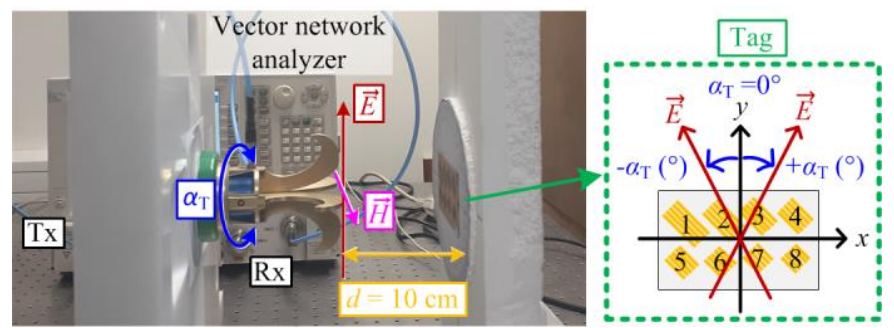

Fig. 2. Measurement setup used in a realistic room environment. $\alpha_{T}$ is the reader interrogation signal angle.

In frequency coded chipless RFID tags, ID is generally coded with the position of the peak apex (extracted from the magnitude of the backscattered signal), which are supposed to correspond to the frequency of resonance $f_{r i}$ of $i$ th scatterer [36]. Each peak apex is expected to appear in resolution frequency slot $d f=100 \mathrm{MHz}$ within the bandwidth of a coding channel $\Delta f=500 \mathrm{MHz}$. Resolution frequency slot $d f$ is an error margin bandwidth of peak apex around its nominal position. As explained in Section I, these peak apexes are aspect-dependent quantities that are affected by the operating environment. So, in a realistic environment, these peak apexes do not occur precisely at the positions of $f_{r i}$. These uncontrolled shifts of peak apexes imply that the bandwidth of the coding channel associated with each scatterer must be enlarged to compensate for these variations of the peak apexes. This action is eventually reducing the coding capacity. Otherwise, a decoding error might happen in the reading process. One solution to reduce the decoding error is to use background normalization. Even so, the background normalization would not aid in the detection of tags in practical cases with mobile objects in the background, where the background is changing instantly or if the tag is attached to 
an unknown object. To show such changes in the reading process, for example, Fig. 3 shows the calibrated $\left|S_{21}\right|$ for REP based chipless tag (see inset of Fig. 2) measured in a realistic room environment at three reader interrogation signal angles $\alpha_{T}: 0^{\circ}, 15^{\circ}$, and $-15^{\circ}$. For the background normalization, the empty measurement (i.e., measurement in the absence of the tag) is measured at $\alpha_{\mathrm{T}}=0^{\circ}$. In the inset of Fig. 3, zoom for the third scatterer of REP chipless tag is presented. As $\alpha_{T}$ changes from $15^{\circ}$ to $-15^{\circ}$ (i.e., reading error due to changes in the environment), the shift between the peak apexes is around $19 \mathrm{MHz}$, while the shift between the dips is $82 \mathrm{MHz}$. This happens due to the superposition of antenna mode and structural mode, which will be explained later in this paper in Section IV. Thus, the peak apexes of signals with slight reading error do not correspond precisely to $f_{r i}$ of the scatterers, as these responses still contain the aspect-dependent information: the coupling and the structural mode (i.e., direct reflections).

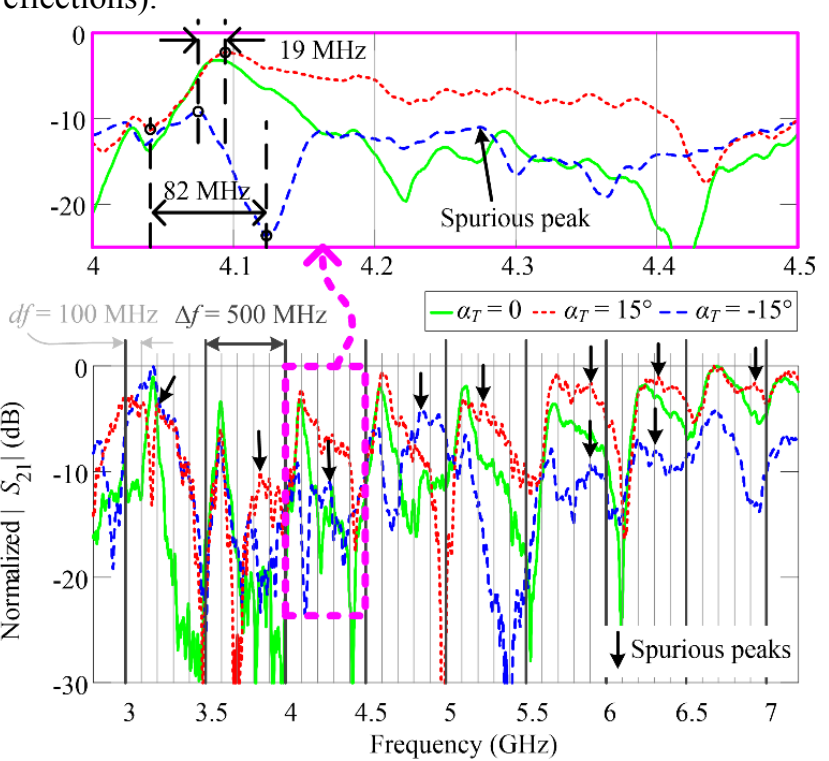

Fig. 3. The calibrated responses $\left|S_{21}\right|$ for REP chipless tag measured in realistic room environment at three reader interrogation signal angles $\alpha_{\mathrm{T}}: 0^{\circ}, 15^{\circ}$, and $-15^{\circ}$. Inset: zoom for the third scatterer of REP chipless tag showing the shifts in the peak apexes and the dips.

Apart from the shifts in the peak apexes, the emergence of spurious peak apexes within each $\Delta f$ can also be observed. Such spurious peak apexes can cause significant decoding errors because some spurious peak apexes exhibit larger magnitude than the actual peak apexes. For this reason, postprocessing techniques are required to extract the aspectindependent parameters for robust detection.

In this paper, we will show that the spurious peak apexes can be ignored by calculating the quality factors (i.e., alternatively damping factors). For this purpose, the initial guess of the quality factor within each $\Delta f$ must be known. In a tag reading process, the general design of target tag is always known that can provide specific a priori information. From our knowledge, in practice, the best way to discard the aspectdependent information and then to extract a priori information is by considering the following conditions:

- The signals are measured in an anechoic environment with a VNA-based chipless RFID reader, where the measurement setup is precisely the same as shown in Fig. 2, but inside an anechoic environment.

- The signals are postprocessed by using background normalization (where both tag and empty measurements are done without changing the reader configuration) and time windowing [to discard the early time response that exhibits a low signal to noise ratio (SNR)].

Table II presents the extracted a priori parameters associated with each scatterer of REP chipless RFID tag (see the inset of Fig. 2). The positions of the peak apexes of signal processed by the above-discussed procedure are used as reference a priori frequencies of resonances $f_{r i}^{\text {ap }}$. The reference $a$ priori quality factors $Q_{i}^{\text {ap }}$ are extracted by using $Q_{i}^{\mathrm{ap}}=f_{r i}^{\mathrm{ap}} / \mathrm{BW}_{-3 \mathrm{~dB}}$, where $\mathrm{BW}_{-3 \mathrm{~dB}}$ is bandwidth at $-3 \mathrm{~dB}$. The reference a priori damping factors $\sigma_{i}^{\text {ap }}$ are calculated as found in [38]:

$$
\sigma_{i}^{\mathrm{ap}}=\frac{\omega_{r i}^{\mathrm{ap}}}{2 Q_{i}^{\mathrm{ap}}}
$$

where $\omega_{r i}^{\mathrm{ap}}=2 \pi f_{r i}^{\mathrm{ap}}$. These extracted parameters can be considered as the closest to the parameters that can be obtained using simulation results and also to CNRs. In this paper, the extracted parameter $f_{r i}^{\text {ap }}$ is also used for comparison to show the accuracy of tag reading success of the proposed method.

TABLE ॥

Extracted $A$ Priori Parameters Associated With EAch Scatterer OF REP CHIPLESS RFID TAG

\begin{tabular}{ccccccccc}
\hline \hline a priori & \multicolumn{8}{c}{ Scatterers } \\
parameters & 1 & 2 & 3 & 4 & 5 & 6 & 7 & 8 \\
\hline$f_{r i}^{\text {ap }}(\mathrm{GHz})$ & 3.18 & 3.69 & 4.19 & 4.69 & 5.23 & 5.79 & 6.29 & 6.77 \\
$Q_{i}^{\text {ap }}$ & 82.42 & 97.1 & 87.66 & 61.93 & 61.41 & 43.92 & 43.74 & 38.32 \\
$\sigma_{i}^{\text {ap }}\left(\times 10^{8}\right)$ & 1.21 & 1.19 & 1.50 & 2.38 & 2.67 & 4.14 & 4.52 & 5.55 \\
\hline \hline
\end{tabular}

\section{PRINCIPLE OF EXTRACTION OF ASPECT-INDEPENDENT PARAMETERS}

Using the SEM theory, the backscattered field of an unknown radar target can be modeled as:

$$
H(s)=\frac{R_{1}}{s-s_{1}}+F(s)
$$

then $s_{1}=-\sigma_{1}+j \omega_{1}\left(\sigma_{1}\right.$ is the damping factor and $\omega_{1}$ is the angular frequency) is CNR of a second order pole. As previously explained, CNRs are aspect-independent information which means that these are the key parameters for target identification. $R$ denotes the residue. For a radar target exhibiting sufficiently large quality factor $Q$, the simplified expressions of $\omega_{1}, \sigma_{1}$ and $R_{1}$ are $\omega_{1} \approx \omega_{0}, \sigma_{1}=\frac{\omega_{0}}{2 Q}$, and $R_{1}=\sigma_{1}\left(1+j \frac{1}{2 Q}\right) . \omega_{0}$ corresponds to the natural frequency. For $R_{1}$, the angle $\theta_{1}$ is $\theta_{1}=\tan ^{-1} \frac{1}{2 Q}$. In (2), the first summand is related to the resonant nature of the target and the second summand $F(s)$ is, in chipless RFID, a non-essential part of the response that might be the structural mode of the target.

For (2), the inverse Laplace transform can be calculated. 


$$
h(t)=2\left|R_{1}\right| u(t) e^{-\sigma_{1} t} \cos \left(\omega_{1} t+\theta_{1}\right)+f(t)
$$

where $h(t)$ is a time domain (TD) response that is constituted of a damped sinusoid including a summand for an uncharacterized response $f(t)$, that is related to structural mode. $u$ corresponds to the unit step function.

Similarly, the transfer function for a multi resonant target can be modeled as the following expression:

$$
H(s)=\sum_{i=1}^{M} \frac{R_{i}}{s-s_{i}}+F(s)
$$

where $M$ is the number of resonances. $s_{i}=\sigma_{i}+j \omega_{i}$ is CNR associated with $i$ th resonance. In a CNR, $\sigma_{i}$ is the damping factor associated with $i$ th resonance and $\omega_{i}$ is the angular frequency associated with $i$ th resonance.

Then, TD backscattered field from a target based on multiresonances can be written as a sum of damped sinusoids including a summand for an uncharacterized response $f(t)$ :

$$
h(t)=\sum_{i=1}^{M} 2\left|R_{i}\right| u(t) e^{-\sigma_{i} t} \cos \left(\omega_{i} t+\theta_{i}\right)+f(t)
$$

Let us now consider chipless RFID applications, and how in such applications, it is possible to extract aspect-independent information from the tag's measurement. To do that, the entire measurement system must be taken into consideration, that is to say, the transmitting and receiving paths, and the surrounding objects' response. To illustrate linear and systematic errors arising in a polarimetric measurement, a block diagram of the channel model was developed in [33], [36]. Note that the antennas effect is taken into account in the transmitting and receiving paths. It is also important to note that due to the presence of the uncharacterized response $f(t)$, in most cases, it is not possible to extract $\omega_{i}$ and $\sigma_{i}$ from the backscattered signal. Indeed, antennas' effect or the surrounding objects' response is most often higher in amplitude than the contribution of the tag. This is all the truer when it is not possible to obtain a calibrated signal. Thus as illustrated in Section II, at best (in a favorable environment, that is to say, an environment without objects outside the tag with known antennas) it is possible to detect the peak apexes of the modulus of the backscattered signal and to associate them to the resonant frequencies of the tag.

To solve this issue, it has been discussed in [33] that by applying the time windowing to the backscattered TD signal from a depolarizing chipless tag (like tags introduced in [36]), the backscattered signal measured by the reader can be approximated as:

$$
\begin{gathered}
h(t) \approx A u\left(t-2 \tau_{r}\right) e^{-\sigma\left(t-2 \tau_{r}\right)} \cos \left(\omega_{0}\left(t-2 \tau_{r}\right)\right) \\
T_{\mathrm{LB}} \leq t \leq T_{\mathrm{LE}}
\end{gathered}
$$

where (6) corresponds to the late time response of chipless tag. $A$ determines the amplitude that depends on the coupling coefficient between emitted pulse and tag, as well as antennas effects. $\tau_{r}$ is the free-space propagation delay on the distance from antenna to tag. $T_{\mathrm{LB}}$ and $T_{\mathrm{LE}}$ are the beginning and ending time of the time window. This result has been obtained based on the block diagram of the channel model, and by using a simplified model for the time domain behavior of each block. In the same way, the approximation of antenna mode for multi-scatterers based tag can be written as:

$$
\begin{gathered}
h(t) \approx \sum_{i=1}^{M} A_{i} u\left(t-2 \tau_{r}\right) e^{-\sigma_{i}\left(t-2 \tau_{r}\right)} \cos \left(\omega_{i}\left(t-2 \tau_{r}\right)\right) \\
T_{\mathrm{LB}} \leq t \leq T_{\mathrm{LE}}
\end{gathered}
$$

Hence, by making the analogy between (5) and (7), the significant result is that this approach, based on depolarizing tags, allows extracting $\omega_{i}$ and $\sigma_{i}$ from the backscattered signal in real scenario, that is to say, the aspect-independent information of the tag.

Note that the choice of $T_{\mathrm{LB}}$ is made after the time instant exhibiting sharp peak apex in TD backscattered signal. This sharp peak apex is associated with the structural mode. Then, $T_{\mathrm{LE}}$ is selected such that the time duration of the time window is sufficient to extract the tag mode. According to [26], at least five cycles of each pole is enough.

Note also that the surrounding objects can be resonant, but we assume that their corresponding frequencies of resonances are not inside the frequency range of operation, and their corresponding quality factors are minimal as compared to quality factor $Q$ of the target we want to identify.

As discussed in [33], the use of a spectrogram representation of the backscattered signal is a compelling way of implementing the time windowing approach. It is why, for the rest of the paper, we will call it spectrogram method.

Finally, to conclude this part, it can be observed that the spectrogram method (7) and SEM (5) reflect the same information as CNRs (i.e., $f_{r i}$ and $\sigma_{i}$ ). For the first time, the accuracy of the extracted parameters using the spectrogram method would be compared to the accuracy of the extracted CNRs using MPM later in this paper.

\section{A. Extraction with Spectrogram Method}

Next, the general procedure of the spectrogram method for employed chipless tags is explained. The basic definition of STFT of a given signal $s(t)$ can be found in [39], [40] as:

$$
S(\omega, \tau)=\int_{-\infty}^{+\infty} s(t) w(\tau-t) e^{-j \omega t} d t
$$

where $\omega=2 \pi f$ is the angular frequency, $w(t)$ is a weighting function or time window and $\tau$ is the time across sliding time window $w(t)$. The effect of the length of the window $w(t)$ to the tradeoff of the resolution between time and frequency has been discussed in [33].

Fig. 4(a) shows the calculated STFT for REP chipless RFID tag measured at $\alpha_{\mathrm{T}}=20^{\circ}$ in a realistic room environment (see Fig. 2). This measurement is chosen particularly to show the robustness of the proposed method because this measurement exhibits multiple spurious peak apexes. The uncalibrated frequency domain (FD) signal measured from VNA is transformed into the uncalibrated TD signal by using inverse fast Fourier transform (IFFT). The uncalibrated TD signal (truncated at $100 \mathrm{~ns}$ ) is supplied to the spectrogram method algorithm, where a hamming window of $16 \mathrm{~ns}$ with $99 \%$ overlap is used to compute STFT. The hamming window of $16 \mathrm{~ns}$ is found the best choice for employed chipless RFID 
tags.

In this proposed spectrogram method, we used multiple averaging windows $\mathrm{AvgW}_{i}$ dedicated to each scatterer in contrast to one averaging window used in [33]. These AvgW $i$ are limited in both frequency bandwidth and time duration [see the inset of Fig. 4(a)]. The bandwidth of each $\operatorname{AvgW}_{i}$ is chosen equal to coding channel bandwidth $\Delta f_{i}$, as each $\Delta f_{i}$ is dedicated to each $i$ th scatterer in FD coded chipless RFID technology. To calculate the time duration of each averaging window $T_{\mathrm{avgW} i}$, the beginning time $T_{\mathrm{avgB} i}$ of each $T_{\mathrm{avgW} i}$ can be defined with respect to $\tau$ of $w(t)$ used in the calculation of STFT. This $T_{\text {avgB } i}$ can be defined as the first-time instant of $\tau$ or soon after a few early time instants of $\tau$ to avoid coupling [33]. Here, we have chosen $T_{\mathrm{avgB} i}$ equal to the first-time instant of $\tau$. The ending time $T_{\mathrm{avgE} i}$ of each $T_{\mathrm{avgW} i}$ is calculated from the decaying envelope generated by using a priori extracted damping factors $\sigma_{i}^{\text {ap }}$ (see Table II):

$$
a_{\mathrm{en} i}=e^{-\sigma_{i}^{\mathrm{ap}} t_{\mathrm{en} i}}
$$

where $a_{e n i}$ and $t_{\mathrm{en} i}$ are the amplitude and time of the decaying envelope.

Then, (9) can be rearranged as

$$
t_{\mathrm{en} i}=\frac{\ln \left(a_{\mathrm{en} i}\right)}{-\sigma_{i}^{\mathrm{ap}}}
$$

By fixing $a_{\mathrm{en} i}$ to a threshold value $a_{\mathrm{en} i}=10^{-3}$ in (10), it is certain that the envelope has sufficiently decayed. So, the ending time of $T_{\mathrm{avgW} i}$ is taken as:

$$
T_{\mathrm{avgE} i}=\left.t_{\mathrm{en} i}\right|_{a_{\mathrm{en} i}=10^{-3}}
$$

Then, the column vectors of $\mathrm{AvgW}$ are averaged. $f_{r i}$ related to $i$ th scatterer is extracted from the peak apexes of STFT averaged $\left(\mathrm{STFT}_{\mathrm{avg} i}\right.$ ) signal above $-3 \mathrm{~dB}$ level. This $-3 \mathrm{~dB}$ value is chosen to select only the significant peak apexes.

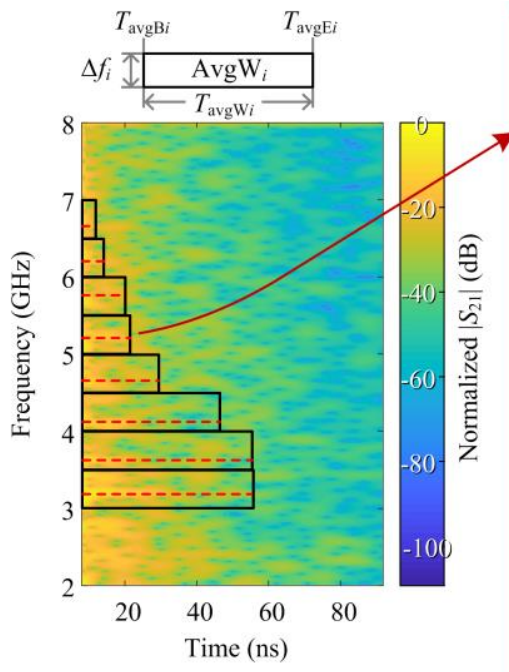

(a)

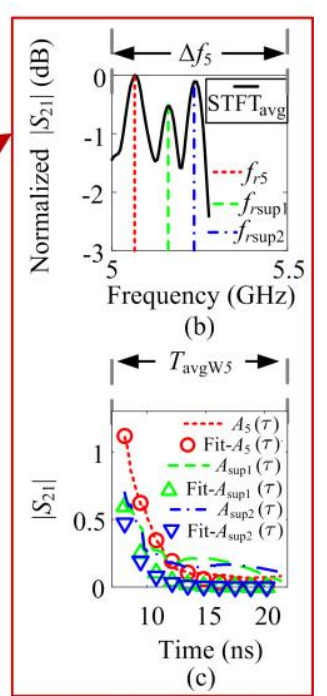

(c)
Fig. 4. Extraction of CNR by spectrogram method using uncalibrated signal measured in a realistic environment. (a) The calculated STFT and multiple dedicated averaging windows AvgW $i$ limited in both frequency bandwidth and time duration. Detection of peak apexes from STFT $_{\text {avgi }}$ above $-3 \mathrm{~dB}$ level and then (b) extraction of $f_{r i}$ based on the (c) extraction of $\sigma_{i}$ from the damping time signal $A_{i}(\tau)$ selected at each peak apex inside $T_{\text {avgwi }}$.
In this spectrogram method, we propose that the spurious peak apexes in $\mathrm{AvgW}_{i}$ can be avoided by calculating the damping factor $\sigma_{i}$ from the decaying time signal $A_{i}(\tau)$ residing inside $\mathrm{AvgW}_{i}$ at all the peak apexes in $\mathrm{STFT}_{\text {avgi }}$ signal. In each $\operatorname{AvgW}_{i}$, the peak apex presenting $\sigma_{i}$ closest to $\sigma_{i}^{\text {ap }}$ are chosen as $f_{r i}$. For example, Fig. 4(b) and (c) show the extraction of $f_{r 5}$ and $\sigma_{5}$. The calculation of $\sigma_{i}$ related to $i$ th scatterer is carried out by the least square method of solving the overdetermined linear system of equations of exponential model $A_{i}(\tau)=A_{o}$ $\exp \left(\sigma_{i} \tau\right)$ for amplitude $A_{o}$ and damping factor $\sigma_{i}$. From Fig. 4(b), it can be observed that the multiple spurious peak apexes $f_{r \text { sup } 1}$ and $f_{r \text { sup2 }}$ along with the correct peak apex $f_{5}$ can emerge. In such a case, the choice of the correct peak apex is made based on the extracted values $\sigma_{i}$ from the damping time signals $A_{i}(\tau)$. The extracted values are $\sigma_{5}=5.76 \times 10^{8}$, $\sigma_{\text {sup } 1}=6.42 \times 10^{8}$, and $\sigma_{\text {sup } 2}=6.62 \times 10^{8}$ for $A_{5}(\tau)$, $A_{\text {sup } 1}(\tau)$, and $A_{\text {sup } 2}(\tau)$, respectively. From these extracted values, $\sigma_{5}$ is closer to the a priori information $\sigma_{5}^{\mathrm{ap}}=2.67 \times$ $10^{8}$. For this reason, in Fig. 4(c), two spurious peak apexes $f_{r \text { sup } 1}$ and $f_{r \text { sup } 2}$ are ignored.

The extracted parameters are $f_{r 5}=5.06 \mathrm{GHz}$ and $Q_{5}=28.62$ which are closer to the extracted a priori parameters $f_{r 5}^{\text {ap }}=$ $5.23 \mathrm{GHz}$ and $Q_{5}^{\mathrm{ap}}=61.41$.

For the rest of this paper for the proposed spectrogram approach, the selected position of the peak apex based on damping factor $\sigma_{i}$ (alternatively quality factor) and the frequency of resonance $f_{r} i$ corresponding to each scatterer will be used interchangeably.

Finally, a second order bandpass filter model is used for the reconstruction of signals as discussed in [41]:

$$
H(\omega)=\sum_{i=1}^{M} \frac{\frac{2 \sigma_{i} j \omega}{\omega_{i}^{2}}}{1+\frac{2 \sigma_{i} j \omega}{\omega_{i}^{2}}+\left(\frac{j \omega}{\omega_{i}}\right)^{2}}
$$

where $\omega_{i}=2 \pi f_{r i}$. M is the number of resonances. The extracted $f_{r i}$ and $\sigma_{i}$ parameters by the proposed spectrogram method (presented next in Fig. 5) are analogous to CNRs.

It is realistic to imagine that the type of to-be-detected tag is known and $\sigma_{i}^{\text {ap }}$ can help in deciding the actual peak apex and discarding the spurious peak apexes within each coding channel $\Delta f_{i}$. Even if the frequency code of a specific scatterer will be different within each coding channel $\Delta f_{i}$ (with a reduction in the metal strip), $\sigma_{i}^{\text {ap }}$ might slightly change. However, in such a case too, it is still helpful in ignoring the spurious peak apexes.

\section{B. Extraction with Matrix Pencil Method}

The algorithm of MPM for calculating the complex poles and residues is explained in [24]. For the implementation of total-least-squares MPM, a data matrix [Y] (see [24, eq. (16)]) is formed by using TD signal. The singular values of the data [Y] are then used to define the desired number of poles $M$ by comparing the ratio of each singular value $\delta$ to the largest singular value $\delta_{\max }$, to a threshold value: 


$$
\frac{\delta}{\delta_{\max }}=10^{-p}
$$

where $p$ is the number of significant decimal digits. In this paper, we have used $p=3$ to generate enough $M$.

First, the uncalibrated FD signal measured from VNA is transformed to the uncalibrated TD signal by using IFFT. Then, a time windowing procedure is applied to extract the sufficient late time response by using a window with the beginning of late time $T_{\mathrm{LB}}$ chosen right after the sharp peak apex in TD signal. Then, the subsequent time duration of $14 \mathrm{~ns}$ is chosen as the width of late time window $T_{\mathrm{LW}}=14 \mathrm{~ns}$. For $r$ $=10 \mathrm{~cm}$ and $\alpha_{\mathrm{T}}=0$, the beginning of late time is $T_{\mathrm{LB}} \approx 1 \mathrm{~ns}$ and the ending of late time is $T_{\mathrm{LE}} \approx 15 \mathrm{~ns}$. Such a choice of $T_{\mathrm{LB}}$ makes the time window selection independent from $r$. This extraction of late time is carried out to discard the early time that is due to the direct reflections from the tag and its holder (the structural mode). Note that early time elimination is mandatory to obtained accurate results. Windowed TD signal is supplied to MPM for the poles' extraction. After extracting the poles, the filtering of the poles is carried out:

1) The poles with positive damping factors $\sigma$ (i.e., exponentially increasing envelopes) are discarded.

2) The poles that do not exhibit their corresponding complex conjugates are discarded. Further, the complex conjugate poles are also discarded and merely the poles with positive $\omega$ are kept.

3) If multiple poles fall in a specific $\Delta f_{i}$ : (a):

the pole with closest $\sigma_{i}$ to $\sigma_{i}^{\text {ap }}$ is selected. This filtering is the same as applied in the spectrogram method. (b):

the poles are filtered out with strict filtering bandwidth of $100 \mathrm{MHz}$, $\mathrm{BW}_{100 \mathrm{MHz}}$, in a range of $50 \mathrm{MHz} \leq f_{r i}^{\text {ap }} \leq 50 \mathrm{MHz}$ instead of step 3-a.
It is important to note that the key difference between the implementation of MPM in this paper and [25] is the pole filtering procedure.

For the uncalibrated signal measured at $\alpha_{T}=0^{\circ}$ in a realistic room environment, the aspect-independent parameters are extracted using the spectrogram method and MPM with poles filtering step 3-a and step 3-b. The signal measured at $\alpha_{T}=0^{\circ}$ is chosen to first compare the performance of both methods without any aspect angle. Fig. 5 shows a comparison of reconstructed FD responses from the spectrogram method and MPM with filtering step 3-a and step 3-b along with the uncalibrated response. Spectrogram method has successfully decoded the tag, while MPM with poles filtering step 3-a remained unsuccessful, as its numerous poles fall out of their known resolution frequency slots $d f_{i}^{\text {ap }}$. On the other hand, MPM with poles filtering step 3-b is successful. A zoom for $7^{\text {th }}$ scatterer is presented in the inset of Fig. 5.

It is very important to mention that the application of MPM is not direct because it needs some skills such as choice of $T_{\mathrm{LB}}$, $T_{\mathrm{LE}}, p$. We have tried our best to select suitable parameters for getting the full performance of MPM. However, for the uncalibrated signals, the decoding inaccuracies of MPM with poles filtering step 3-a are very frequent. For MPM, the poles filtering step 3-b is not realistic, as $f_{r i}^{\text {ap }}$ is dependent on the tag code. We have applied such strict filtering (poles filtering step 3-b) to provide better information about the extraction with MPM.

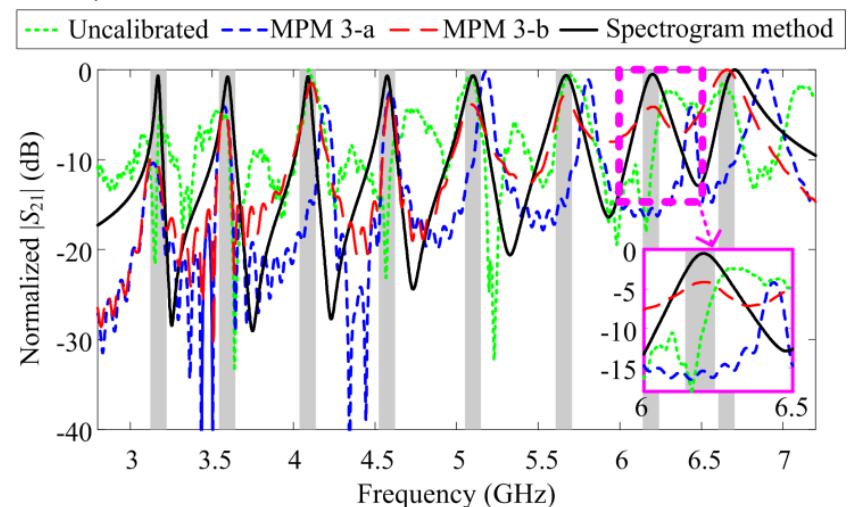

Fig. 5. Comparison of spectrogram method and MPM along with uncalibrated signal. Shaded areas are in the range of $-50 \mathrm{MHz} \leq f_{r i}^{\mathrm{ap}} \leq$ $50 \mathrm{MHz}$.

\section{EXTRACTION OF THE Aspect-InDEPENDENT TAG Code}

In this Section, first, an analytical description of various kinds of superpositions between the tag mode and the structural mode is presented. An example of such superpositions is explained above in the inset of Fig. 3. Then, the extractions of tag code in various realistic configurations are presented.

The response of the second order scatterer exhibits a peak apex along with a dip, where the peak apex and dip occur due to the in-phase superposition and out-of-phase superposition of the tag's antenna mode and the structural mode, respectively. It has been discussed in [42] that the time windowing (or gating) is a solution to remove part of the structural mode from the response, thus to obtain only one peak apex. This new peak apex should be close to the nominal peak apex but more precisely between the nominal peak apex and the dip. To model both the tag mode and the structural mode of second order scatterer tag, a transfer function based on two bandpass filters can be used. For the sake of clarity and to introduce the notations, (12) is rewritten as:

$$
H(\omega)=\frac{G_{\mathrm{sc}} \cdot \frac{2 \sigma_{\mathrm{sc}} j \omega}{\omega_{\mathrm{sc}}^{2}} \cdot e^{-j \phi_{\mathrm{sc}}}}{1+\frac{2 \sigma_{\mathrm{sc}} j \omega}{\omega_{\mathrm{sc}}^{2}}+\left(\frac{j \omega}{\omega_{\mathrm{sc}}}\right)^{2}}+\frac{G_{\mathrm{st}} \cdot \frac{2 \sigma_{\mathrm{st}} j \omega}{\omega_{\mathrm{st}}^{2}} \cdot e^{-j \phi_{\mathrm{st}}}}{1+\frac{2 \sigma_{\mathrm{st}} j \omega}{\omega_{\mathrm{st}}^{2}}+\left(\frac{j \omega}{\omega_{\mathrm{st}}}\right)^{2}}
$$

where, $\phi_{\mathrm{sc}}, \omega_{\mathrm{sc}}$ and $\sigma_{\mathrm{sc}}$ correspond to the phase, angular frequency of resonance and damping factor of the scatterer, respectively. $\phi_{\mathrm{st}}, \omega_{\mathrm{st}}$ and $\sigma_{\mathrm{st}}$ correspond to the phase, angular frequency of resonance and damping factor of the structural mode, respectively. The gains $G_{\mathrm{sc}}$ and $G_{\mathrm{st}}$ determine the amplitude level of both the tag and the structural modes, respectively. Fig. 6 illustrates the emergence of dip between the mode associated with the scatterer mode sc $_{\text {(solid red line) }}$ generated by first summand of (14) and the mode associated with the structure mode $_{\text {st }}$ (dashed blue line) generated by second summand of (14). From the combined response mode $_{\mathrm{sc}+\mathrm{st}}$ generated by (14), it can be observed that the dip emerges due to the destructive interference between mode $\mathrm{sc}_{\mathrm{sc}}$ 
and mode $_{\text {st }}$ at the point when the phase difference between them is $180^{\circ}$.

To generalize (14) for multi-scatterers tag more summands like first summand of (14) can be added.

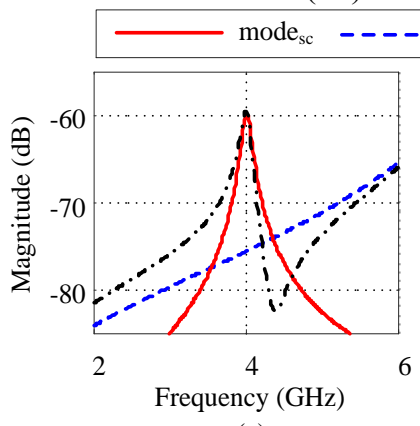

(a)

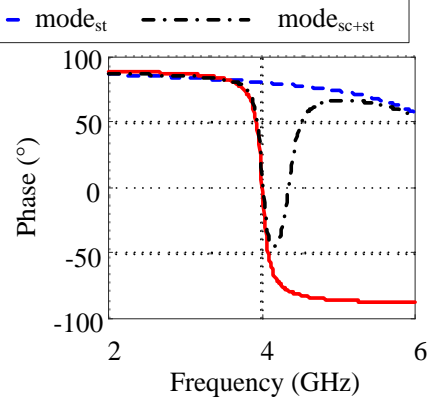

(b)
Fig. 6. Illustration of the destructive interference between the mode associated with the scatterer mode ${ }_{s c}$ generated by the first summand of (14) and the mode associated with the structure mode st $_{\text {generated }}$ by the second summand of (14). (a) Magnitude responses. (b) Phase responses. The parameters used to generate the responses using (14) are: $f_{\mathrm{sc}}=4 \mathrm{GHz}, f_{\mathrm{st}}=7 \mathrm{GHz}, G_{\mathrm{sc}}=G_{\mathrm{st}}=0.001, \sigma_{\mathrm{sc}}=4.4 \times 10^{9}$ (i.e., $\left.Q_{\mathrm{sc}}=30\right)$ and $\sigma_{\mathrm{st}}=4.19 \times 10^{8}$ (i.e., $Q_{\mathrm{st}}=5$ ). Both mode $\mathrm{sc}$ and mode are taken initially in phase thus $\varphi_{\mathrm{sc}}=\varphi_{\mathrm{st}}=0$.

In Fig. 6, the addition of mode st $_{\text {[second summand of (14)] }}$ to mode sc [first summand of (14)] leads to a shift in the peak apex of resultant mode ${ }_{\mathrm{sc}+\mathrm{st}}$ from the peak apex of mode $\operatorname{sc}_{\mathrm{sc}} \mid \Delta f_{\mathrm{sc}}$ to sctst around $6 \mathrm{MHz}$. Thus, variations of the parameters of (14) can produce significant $\left|\Delta f_{\text {sc to sc+st }}\right|$. Fig. 7 presents a parametric study of variations of two parameters of (14): variation of $G_{\text {st }}$ in Fig. 7(a) and variation of $\phi_{\mathrm{sc}}$ in Fig. 7(b). The variation of $G_{\text {st }}$ in (14) with numerical values equal to $0.005,0.01$, and 0.02 is producing $\left|\Delta f_{\text {sc to sc+st }}\right|$ equal to $30.55 \mathrm{MHz}, 37.94 \mathrm{MHz}$, and $40.44 \mathrm{MHz}$, respectively [see Fig. 7(a)]. On the other hand, the variation of $\phi_{\mathrm{sc}}$ in (14) from $0^{\circ}$ to $270^{\circ}$ with a step of $90^{\circ}$ is producing $\left|\Delta f_{\text {sc to sc+st }}\right|$ equal to $30.55 \mathrm{MHz}, 151.92 \mathrm{MHz}$, $46.98 \mathrm{MHz}$, and $4.75 \mathrm{MHz}$, respectively [see Fig. 7(b)]. Symbols $\times$ show the peak apexes.

In Fig. 7(b), it can be observed that as $\phi_{\mathrm{sc}}$ changes from $0^{\circ}$ to $180^{\circ}$, the dip associated with the peak apex shifts from right to left. For this reason, such an aspect-dependent dip cannot be used to encode the tag. A similar phenomenon of various kinds of superpositions can be observed in Fig. 3. Each mode $_{\text {sc }+s t}$ exhibits different types of superposition, where the shifts of the dip associated with the peak apex can also be observed.

\section{A. Extraction of the Orientation-Independent Tag Code}

In practical cases, if the tag is affixed or printed on the tobe-identified object, it might be impossible to detach the tag to take an empty measurement for background normalization. However, even if the background normalization is possible, a change in the orientation of the reader (or orientation of the tag) might lead to uncontrolled shifts in the peak apexes corresponding to $f_{r i}$, as explained experimental measurements in Fig. 3 and by the principle of superpositions in Fig. 7. These shifts in the peak apexes could further impact the extraction of tag code as the depolarizing tags (see the inset in Fig. 2) are coded based on the position of the peak apexes. To circumvent such a challenge, the extraction of the aspect-independent parameters is necessary. Fig. 8 shows the extracted $f_{r i}$ using
MPM with poles filtering step 3-b and spectrogram method for $-20^{\circ} \leq \alpha_{T} \leq-20^{\circ}$ measured in a realistic environment using the measurement setup shown in Fig. 2. The spectrogram method is applied as explained in Section III-A, and MPM is applied with poles filtering step 3-b as explained in Section III-B. It can be observed that the performance of the spectrogram method is better than MPM, as the spectrogram method is successful for all aspect angles $-20^{\circ} \leq \alpha_{T} \leq-20^{\circ}$. MPM with poles filtering step 3-b is unsuccessful at two aspect angles $\alpha_{T}$ $=\left[-20^{\circ}, 10^{\circ}\right]$. Conversely, MPM with poles filtering step 3-a is unsuccessful for all aspect angles $-20^{\circ} \leq \alpha_{T} \leq-20^{\circ}$.

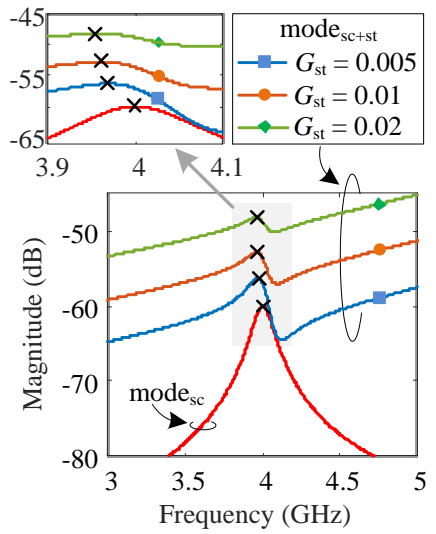

(a)

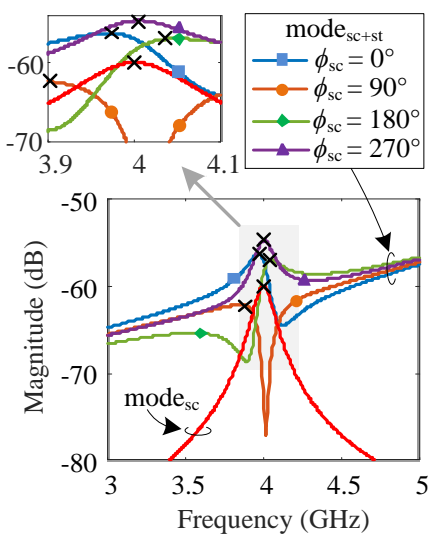

(b)
Fig. 7. Variations in the position of peak apex generated by using (14). (a) Variation due to the change in the gain of structural mode $G_{\text {st }}$, where $G_{\mathrm{sc}}=0.001$ and $G_{\mathrm{st}}=[0.005,0.01,0.02]$. (b) Variation due to the change in the phase of scatterer mode $\phi_{\mathrm{sc}}$, where $G_{\mathrm{sc}}=0.001$, $G_{\text {st }}=0.005, \phi_{\text {st }}=0$ and $\phi_{\text {sc }}=\left[0^{\circ}, 90^{\circ}, 180^{\circ}, 270^{\circ}\right]$. The rest of the parameters in (14) are taken exactly as shown in the caption of Fig. 6. Symbols $\times$ show the peak apexes.

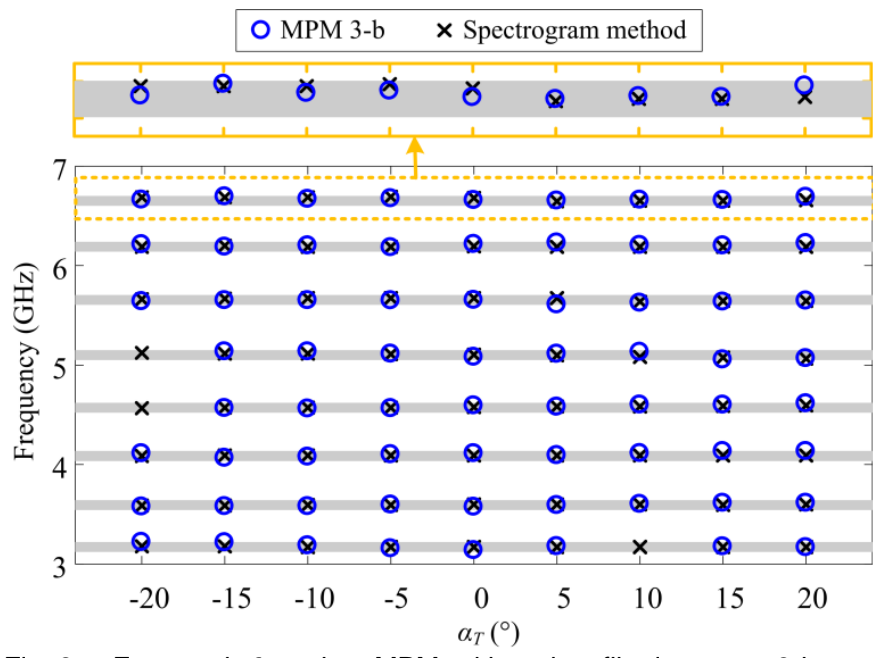

Fig. 8. Extracted $f_{r i}$ using MPM with poles filtering step 3-b and spectrogram method for $-20^{\circ} \leq \alpha_{\mathrm{T}} \leq-20^{\circ}$. Shaded areas are in the range of $-50 \mathrm{MHz} \leq f_{r i}^{\text {ap }} \leq 50 \mathrm{MHz}$.

The variation of extracted $f_{r i}$ in the range $-20^{\circ} \leq \alpha_{\mathrm{T}} \leq-20^{\circ}$ (calculated from the absolute difference between the minimum and maximum values of $f_{r i}$ ) defines resolution frequency $d f_{i}$ for each $i$ th scatterer. The calculated $d f_{i}$ for the spectrogram method is outlined in Table III. 
TABLE III

The Calculated Resolution Frequencies $d f_{i}$ Using Spectrogram METHOD WITH $-20^{\circ} \leq \alpha_{\mathrm{T}} \leq 20^{\circ}$

\begin{tabular}{ccccccccc}
\hline \hline & 1 & 2 & 3 & 4 & 5 & 6 & 7 & 8 \\
\cline { 2 - 9 }$d f_{i}(\mathrm{MHz})$ & 5.86 & 11.72 & 5.86 & 29.3 & 58.6 & 35.16 & 11.72 & 46.88 \\
\hline \hline
\end{tabular}

The number of combinations $N B$ (number of bits - coding capacity) for such depolarizing tags can be calculated by using the expression found in [36]:

$$
N B=\left(\frac{\Delta f}{d f}\right)^{k}
$$

where $\Delta f$ is the bandwidth of coding channel and $d f$ is resolution frequency. In [36], the calculated $N B$ is equal to 390625 (18.5 bits) by using the following values: $\Delta f=500 \mathrm{MHz}, k=8$, and $d f=100 \mathrm{MHz}$. For the spectrogram method, it can be observed in Table III that the worst value of resolution frequency is $d f_{5}=58.6 \mathrm{MHz}$. If we take $d f=60 \mathrm{MHz}$ in (15), a value of 24.4 bits can be calculated. Hence, the proposed spectrogram method shows an improvement of 5.8 bits in the coding capacity of REP chipless RFID tags [see Fig. 1(a)]. Conversely, the improvement achieved by MPM is not realistic because of the strict poles filtering step 3-b.

In terms of the reading interrogation angle $\alpha_{\mathrm{T}}$ (or the misalignment of chipless tag), the minimum detectability limit of the employed chipless tag is $\alpha_{\mathrm{T}}=45^{\circ}$. This is because at $\alpha_{\mathrm{T}}=45^{\circ}$, the employed chipless RFID tag does not behave as a depolarizing scatterer. In such a case, the microstrip coupled dipoles might be aligned vertically or horizontal producing a null cross polarization response $S_{21}$ (by ignoring the backscattered response from the nonsymmetrical shape of the tag, if any).

Next, we have analyzed the minimum detectability limit of the distance between the tag and antenna $d^{\prime}$ and the tag azimuth angle $\theta$. For this purpose, the experimental measurements of the chipless tag are done by varying $d^{\prime}$ and $\theta$ as shown in Fig. 9. The measurement parameters and equipment such as antenna, vector network analyzer (VNA), output power, and frequency sweeping along with the number of sweep points are precisely the same as discussed for the measurement setup shown in Fig. 2. The $d^{\prime}$ is varied from $15 \mathrm{~cm}$ to $40 \mathrm{~cm}$ with a step of $5 \mathrm{~cm}$ and the $\theta$ is changed from $-60^{\circ}$ to $60^{\circ}$ with a step of $10^{\circ}$. For each variation, the spectrogram method and MPM with poles filtering step 3-a are applied. The MPM with poles filtering step 3-a is unsuccessful for all steps. For this reason, Fig. 10 shows only the tag reading performance of the spectrogram method. It can be observed from Fig. Fig. 10 that at $\theta=0^{\circ}$ (i.e., without any aspect angle), the minimum detectability limit of the distance $d_{1}^{\prime}$ is $25 \mathrm{~cm}$ for the employed chipless RFID tag mounted on the Polystyrene foam. For the variation of $\theta$, the $d^{\prime}=15 \mathrm{~cm}$ is most suitable, as the tag is detectable up to $\theta=40^{\circ}$. At $d^{\prime}=$ $20 \mathrm{~cm}$ and $d^{\prime}=25 \mathrm{~cm}$, the detection is successful up to $\theta=-$ $10^{\circ}$ and $\theta=40^{\circ}$, respectively. We have also applied both detection techniques (the spectrogram method and MPM with poles filtering step 3-a) by considering the six first peaks apexes (i.e., six first resonators), due to the lower SNR observed for the higher frequencies for distances $d^{\prime}$ greater than $10 \mathrm{~cm}$. In such a case, the tag detection is also possible at $\theta=60^{\circ}$ for $d^{\prime}=15 \mathrm{~cm}$. For $d^{\prime}=20 \mathrm{~cm}$ and $d^{\prime}=25 \mathrm{~cm}$, the tag is also detected at $\theta=\left[10^{\circ},-20^{\circ}\right]$ and $\theta=10^{\circ}$, respectively.

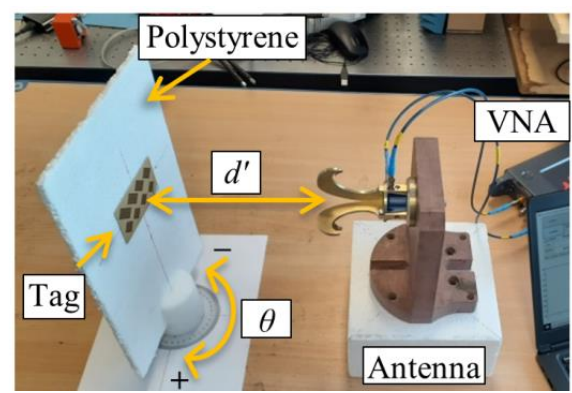

Fig. 9. Measurement setup presenting the tag attached to polystyrene foam in a realistic room environment.

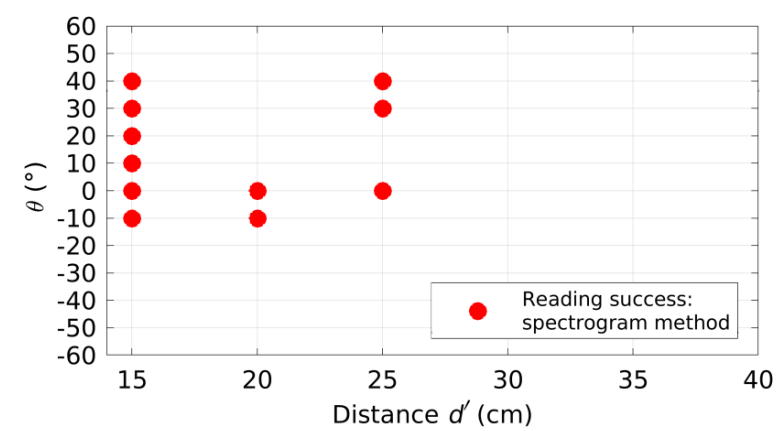

Fig. 10. Measurement setup presenting the tag attached to polystyrene foam in a realistic room environment.

\section{B. Extraction of the Object-Independent Tag Code}

In practical cases, 1) variations in the size of to-be-labeled object might produce variations in the gain of structural mode. 2) irregularity (or asymmetry) of to-be-labeled object might produce a phase difference between the tag mode and the structural mode. 3) variations of the distance of an object from the reader antenna might produce both gain and phase shift. These variations can produce random shifts in the peak apexes of measured response from their nominal positions, which can further lead to a decoding error. Again, to circumvent this challenge, extraction of the aspect-independent parameters is necessary. To explain this challenge, we have done experimental measurements with an asymmetrical dielectric slab of phenolic resin laminate (CARP) behind the chipless tag in a realistic room environment as shown in Fig. 11. The thickness of dielectric slab of CARP is $10 \mathrm{~mm}$. The asymmetry is produced by displacing the slab by $3 \mathrm{~cm}$ from its center. The distance $d$ between the tag and the dielectric slab of CARP is varied from 0 to $5 \mathrm{~cm}$ by keeping dielectric slab fixed at $15 \mathrm{~cm}$ from the reader antenna. The parameter $S_{21}$ is measured at each displacement in a cross-polarization configuration. The rest of the measurement parameters such as VNA, output power, and frequency sweeping along with the number of sweep points are precisely the same as discussed for the measurement setup shown in Fig. 2. It is important to note that we have performed measurements using various kinds of dielectric slabs such as polyurethane, PET, PTFE, and CARP. However, we have chosen the results for a dielectric 
slab of CARP to present in this work due to its high permittivity of 5.7 .

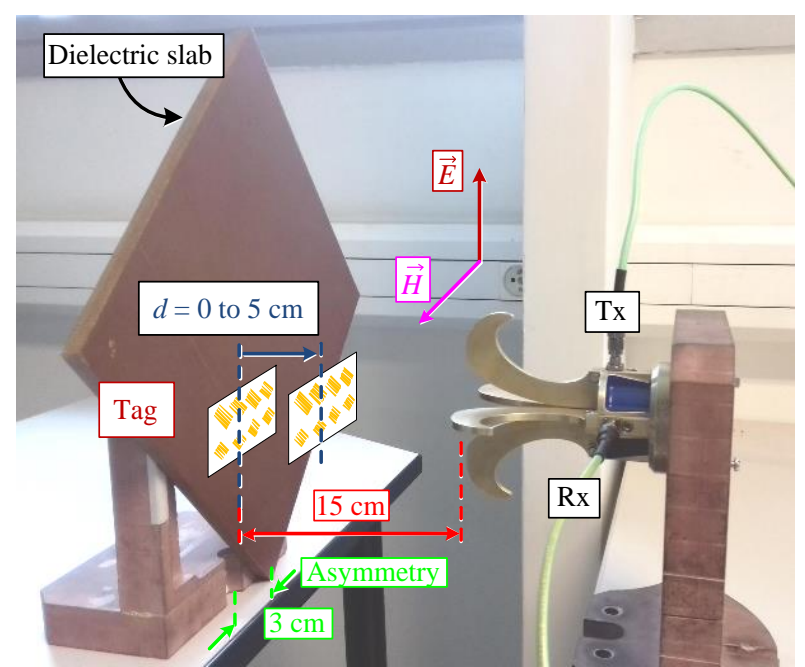

Fig. 11. Measurement setup for varying the distance between the dielectric slab of CARP $\left(\varepsilon_{r}=5.7\right.$ and thickness $\left.=10 \mathrm{~mm}\right)$ and the tag $d$ in a realistic room environment.

Fig. 12 shows the extracted $f_{r i}$ using MPM with poles filtering step 3-b and spectrogram method with $d$ ranging from 0 to $5 \mathrm{~cm}$. The spectrogram method is applied as explained in Section III-A, and MPM is applied with poles filtering step 3$\mathrm{b}$ as explained in Section III-B. It can be observed that the performance of the spectrogram method is better than MPM, as the spectrogram method is successful for all values of distance $d$ from 0 to $5 \mathrm{~cm}$. MPM with poles filtering step 3-b is only successful at two values of $d=[3 \mathrm{~cm}, 4 \mathrm{~cm}]$. Conversely, MPM with poles filtering step 3-a unsuccessful for all values of distance $d$ from 0 to $5 \mathrm{~cm}$. The calculated $d f_{i}$ for spectrogram method is outlined in Table IV.

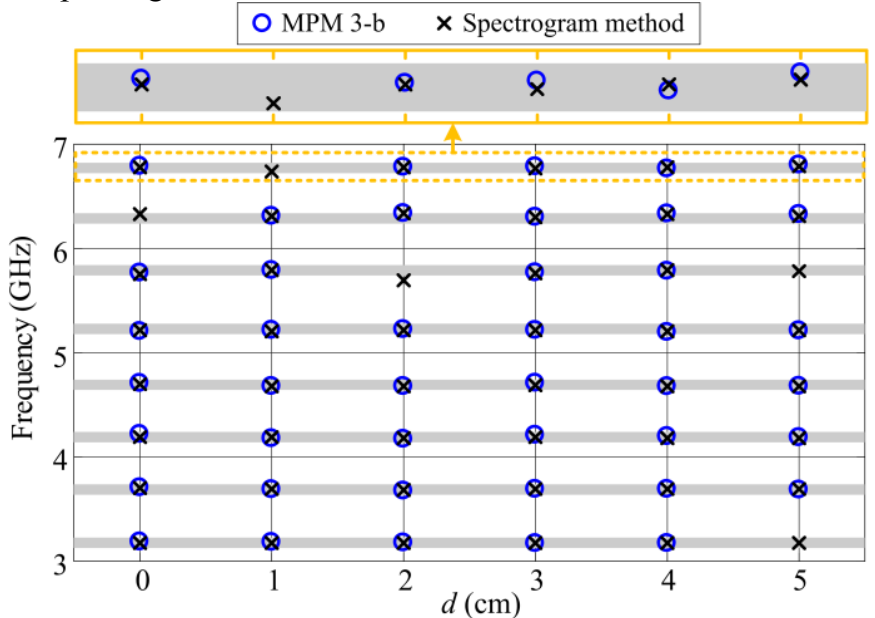

Fig. 12. Extracted $f_{r} i$ using MPM with poles filtering step 3-b and spectrogram method while $d$ is ranging from 0 to $5 \mathrm{~cm}$. Shaded areas are in the range of $-50 \mathrm{MHz} \leq f_{r i}^{\text {ap }} \leq 50 \mathrm{MHz}$.

TABLE IV

THE CalCulated Resolution FREQUencies $d f_{i}$ Using SPECTROgRam METHOD WITH $0 \leq d \leq 5 \mathrm{CM}$

\begin{tabular}{ccccccccc}
\hline \hline & \multicolumn{8}{c}{ Scatterer $i$} \\
\cline { 2 - 9 }$d f_{i}(\mathrm{MHz})$ & 1 & 2 & 3 & 4 & 5 & 6 & 7 & 8 \\
\hline \hline
\end{tabular}

We are limited in terms of the maximum possible dielectric constant and different sizes of the dielectric slabs. Therefore, we have tested the worst possible cases. Fig. 13 shows measurement setups presenting the tag attached to various objects in a realistic room environment: Polystyrene foam spacers [Fig. 13(a)], metallic plate [Fig. 13(b)], spray bottle [Fig. 13(c)], and water tank [Fig. 13(d)]. Here, too, the measurement parameters and equipment are precisely the same as discussed for the measurement setup shown in Fig. 2. The distance between the tag and antenna $d^{\prime}$ is varied from $15 \mathrm{~cm}$ to $40 \mathrm{~cm}$ with a step of $5 \mathrm{~cm}$. For each variation, the spectrogram method and MPM with poles filtering step 3-a are applied. The MPM with poles filtering step 3-a is unsuccessful for all steps. For this reason, Fig. 14 shows only the tag reading performance of the spectrogram method. For the Polystyrene spacers placed between the antenna and tag [see Fig. 13(a)], it can be observed from Fig. 14, the minimum detectability limit of the distance $d_{2}^{\prime}$ is $25 \mathrm{~cm}$. The $d_{2}^{\prime}$ is equal to $d_{1}^{\prime}$ as observed in Fig. 9 at $\theta=0^{\circ}$. For the chipless tag mounted on the metallic plate [see Fig. 13(b)], the minimum detectability limit of the distance $d_{3}^{\prime}$ equal to $20 \mathrm{~cm}$ can be observed in Fig. 14. The $d_{3}^{\prime}<d_{2}^{\prime}$ due to the large reflections from metallic plate. For the chipless tag mounted on the spray bottle [see Fig. 13(c)], the detection of tag is only successful for $d^{\prime}=[15 \mathrm{~cm}, 25 \mathrm{~cm}]$. The minimum detectability limit of the distance $d_{4}^{\prime}$ is $25 \mathrm{~cm}$. For the chipless tag mounted on the water tank [see Fig. 13(d)], the detection of tag is only successful for $d^{\prime}=25 \mathrm{~cm}$. However, if we consider only the six first peak apexes, the detection of tag is also successful for $d^{\prime}=20 \mathrm{~cm}$ for the tag mounted on the spray bottle [see Fig. 13(c)].

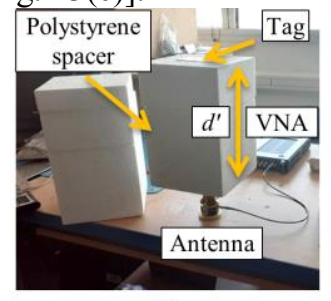

(a)

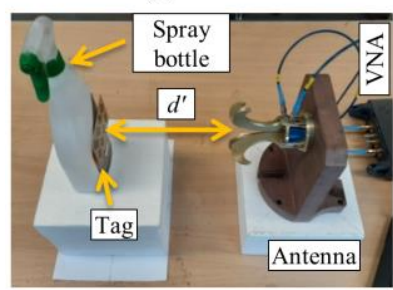

(c)

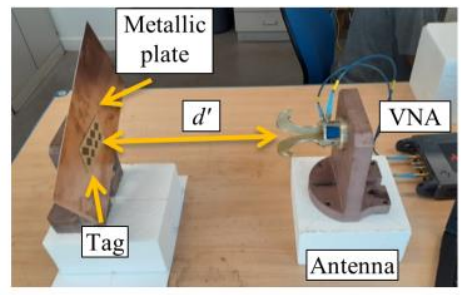

(b)

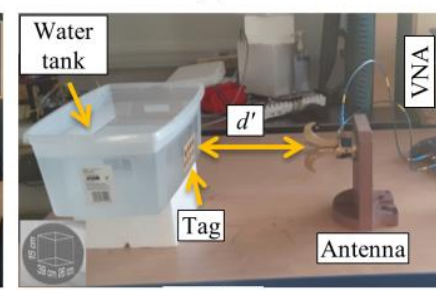

(d)
Fig. 13. Measurement setups presenting the tag attached to various objects in a realistic room environment. (a) Polystyrene foam spacers. (b) Metallic plate. (c) Spray bottle. (d) Water tank.

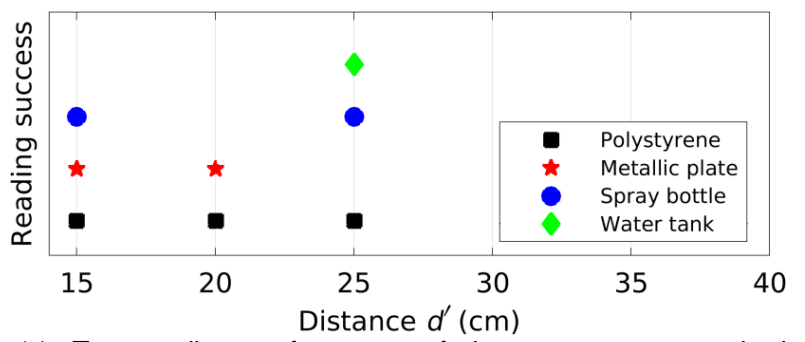

Fig. 14. Tag reading performance of the spectrogram method for measurement setups shown in Fig. 13. 


\section{Extraction of Tag Code on 3-Dimensional Reading Zone}

We have also compared the performance of both spectrogram method and MPM for the signals measured on 3D measurement bench. The measured signals are obtained from the measurements used in [43]. The antenna is fixed and the chipless tag is moving in $3 \mathrm{D}$ volumetric space (i.e., defined as $x \in[-10 ; 10] \mathrm{cm}, y \in[-10 ; 10] \mathrm{cm}$ and $z \in[0.2 ; 20] \mathrm{cm})$ with a step size of $1 \mathrm{~cm}$ for each axial parameter (only the first step for $z$ is from 0.2 to $1 \mathrm{~cm}$ ). More details about the measurement setup can be seen in [43].

The spectrogram method is applied as explained in Section III-A, and MPM is applied with poles filtering step 3-a and 3$\mathrm{b}$ as explained in Section III-B. It is important to note that MPM with pole filtering step 3-b is not realistic in terms of application (as described previously). However, it allows us to illustrate the extraction with MPM.

3D representations of the performance of spectrogram method and MPM with pole filtering step 3-a are shown in Fig. 15. Each point shows the reading success. Additionally, the percentage of reading success area is calculated for each step along $z$ for full 3D characterization of the performance. For spectrogram method, the maximum and minimum reading success is $25.62 \%$ and $4.76 \%$ at $z=10 \mathrm{~cm}$ and $z=20 \mathrm{~cm}$, respectively. Furthermore, for spectrogram method, the reading success in full volume is $15.60 \%$. For MPM with pole filtering step $3-\mathrm{a}$ and $3-\mathrm{b}$, the reading success in full volume is $0.6 \%$ and $23.8 \%$, respectively. Even if we use calibrated signals (i.e., with background normalization) for MPM with pole filtering step $3-a$, the success rate in full volume merely improves to $1.4 \%$.

Finally, we have also implemented the spectrogram method without improvement in the accuracy of extraction of frequency of resonance in each coding channel based on its corresponding quality factor. This implementation is similar to the STFT averaging method presented in [33], except the multiple averaging windows $\mathrm{AvgW}_{i}$ dedicated to each scatterer in contrast to one averaging window. In such an implementation, the emergence of multiple peak apexes (including the desired and spurious peak apexes) in a coding channel results in the unsuccessful decoding of the tag ID. This implementation is referred to as the conventional spectrogram method.

At $y=0,2 \mathrm{D}$ representations of the performance of the proposed spectrogram method and the conventional spectrogram method are shown in Fig. 16(a) and (b), respectively. Each black square with a size of $1 \mathrm{~cm}^{2}$ shows the reading success. At $y=0$, the percentages of reading success area for the proposed spectrogram method [Fig. 16(a)] and the conventional spectrogram method [Fig. 16(b)] are $40.36 \%$ and $6.12 \%$, respectively. For conventional spectrogram method in $3 \mathrm{D}$ volumetric reading space, the maximum and minimum reading success are $4.3 \%$ and 0 at $z=9 \mathrm{~cm}$ and $z=[15,17,18$, $19,20] \mathrm{cm}$, respectively. Furthermore, for conventional spectrogram method, the reading success in full volume is $1.65 \%$. Conversely, at $y=0$, the performance of MPM with filtering step $3-\mathrm{a}$ and $3-\mathrm{b}$ is $0.9 \%$ and $48.1 \%$, respectively. Hence, the proposed spectrogram method has better reading success than conventional spectrogram method and MPM with poles filtering step 3-a (which is comparable to the proposed spectrogram method in terms of implementation).

For spectrogram method, the maximum reading success $(25.62 \%$ at $z=10 \mathrm{~cm})$ and the volumetric reading success $(15.60 \%)$ seem low. This is because of the large 3D volumetric reading space. If we reduce the $3 \mathrm{D}$ volumetric space such that $x \in[-3 ; 3] \mathrm{cm}, y \in[-3 ; 3] \mathrm{cm}$ and $z \in[5$; $15] \mathrm{cm}$, the maximum reading success is $89.79 \%$ at $z=10 \mathrm{~cm}$ and $36.73 \%$ at $z=9 \mathrm{~cm}$ for the proposed spectrogram method and the conventional spectrogram method, respectively. The volumetric reading success is $81.63 \%$ and $18.55 \%$ for the proposed spectrogram method and the conventional spectrogram method, respectively.

In general, the computational time for spectrogram method is faster than MPM, because it is based on fast Fourier transform (FFT).

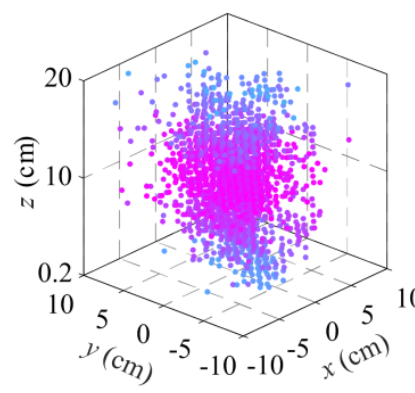

(a)

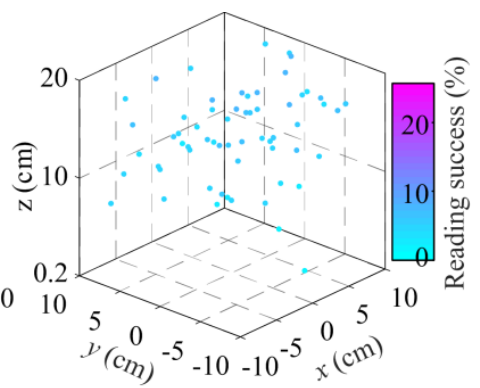

(b)
Fig. 15. 3D representation of reading success at all displacements of z. (a) Proposed spectrogram method. (b) MPM with poles filtering step 3-a.

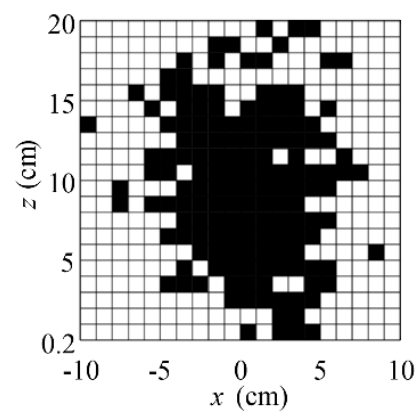

(a)

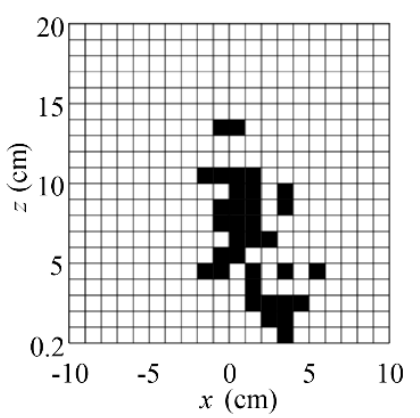

(b)
Fig. 16. Reading performance at $y=0$. (a) Proposed spectrogram method. (b) Conventional spectrogram method.

\section{CONCLUSION}

In this paper, robust detection of the depolarizing REP tags using fast Fourier transform (FFT) based spectrogram method was demonstrated. The robustness of the proposed technique was proved by reading the identification (ID) of the chipless tag: 1) at various misalignments of the reader (and the tag). 2) at various distances for the tag mounted on several objects. 3) at various displacements using $3 \mathrm{D}$ measurements. We have demonstrated that for frequency-coded chipless RFID technology, the spectrogram method is an efficient and possibly fast choice. The extraction of complex natural frequency(ies) using the spectrogram was never done earlier in the field of frequency-coded chipless RFID. With an operation of a single measurement, the proposed technique is very 
promising for the practical implementation of the chipless RFID technology, as the tags ID can be decoded accurately using the extracted quality factor information. Furthermore, the proposed technique is computationally less expensive due to the inherent fast property of FFT. Thus, the proposed technique requires fewer resources and effort.

\section{REFERENCES}

[1] E. Perret, Radio Frequency Identification and Sensors: From RFID to Chipless RFID. John Wiley \& Sons, 2014.

[2] E. M. Amin, M. S. Bhuiyan, N. C. Karmakar, and B. Winther-Jensen, "Development of a Low Cost Printable Chipless RFID Humidity Sensor," IEEE Sensors J., vol. 14, no. 1, pp. 140-149, Jan. 2014.

[3] Y. Feng, L. Xie, Q. Chen, and L. Zheng, "Low-Cost Printed Chipless RFID Humidity Sensor Tag for Intelligent Packaging," IEEE Sensors J., vol. 15, no. 6, pp. 3201-3208, Jun. 2015.

[4] M. Borgese, F. A. Dicandia, F. Costa, S. Genovesi, and G. Manara, "An Inkjet Printed Chipless RFID Sensor for Wireless Humidity Monitoring," IEEE Sensors J., vol. 17, no. 15, pp. 4699-4707, Aug. 2017.

[5] A. Vena, E. Perret, D. Kaddour, and T. Baron, "Toward a Reliable Chipless RFID Humidity Sensor Tag Based on Silicon Nanowires," IEEE Trans. Microw. Theory Techn., vol. 64, no. 9, pp. 2977-2985, Sep. 2016.

[6] A. Vena, L. Sydänheimo, M. M. Tentzeris, and L. Ukkonen, "A Fully Inkjet-Printed Wireless and Chipless Sensor for CO2and Temperature Detection," IEEE Sensors J., vol. 15, no. 1, pp. 89-99, Jan. 2015.

[7] H. E. Matbouly, S. Tedjini, K. Zannas, and Y. Duroc, "Compact Multi Bit Slotted C-Scatterer for Threshold Sensitive Chipless Wireless Temperature Sensor," Technol., vol. 6, no. 3, 2018.

[8] F. Lu, H. Wang, Y. Guo, Q. Tan, W. Zhang, and J. Xiong, "Microwave Backscatter-Based Wireless Temperature Sensor Fabricated by an Alumina-Backed Au Slot Radiation Patch," Sensors, vol. 18, no. 1, 2018.

[9] A. Guillet, A. Vena, E. Perret, and S. Tedjini, "Design of a chipless RFID sensor for water level detection," in Proc. International Symposium on Antenna Technology and Applied Electromagnetics, Jun. 2012, pp. 1-4.

[10] E. Perret, "Permittivity characterization based on Radar Cross measurements," in Proc. URSI International Symposium on Electromagnetic Theory (EMTS), Aug. 2016, pp. 457-460.

[11] F. Costa et al., "A Depolarizing Chipless RF Label for Dielectric Permittivity Sensing," IEEE Microw. Wireless Compon. Lett., vol. 28, no. 5, pp. 371-373, 2018.

[12] A. Lázaro et al., "Chipless Dielectric Constant Sensor for Structural Health Testing," IEEE Sensors J., vol. 18, no. 13, pp. 5576-5585, 2018, doi: 10.1109/JSEN.2018.2839689.

[13] A. Vena, M. Tedjini, T. Björninen, L. Sydänheimo, L. Ukkonen, and M. M. Tentzeris, "A novel inkjet-printed wireless chipless strain and crack sensor on flexible laminates," in Proc. IEEE Antennas and Propagation Society International Symposium (APSURSI), Jul. 2014, pp. 1294-1295.

[14] A. M. J. Marindra and G. Y. Tian, "Chipless RFID Sensor Tag for Metal Crack Detection and Characterization," IEEE Trans. Microw. Theory Techn., vol. 66, no. 5, pp. 2452-2462, May 2018, doi: 10.1109/TMTT.2017.2786696.

[15] E. Perret, "Displacement Sensor Based on Radar Cross-Polarization Measurements," IEEE Trans. Microw. Theory Techn., vol. 65, no. 3, pp. 955-966, Mar. 2017.

[16] N. Barbot and E. Perret, "Gesture recognition with the chipless RIFD technology," in Proc. XXXIInd General Assembly Scientific Symp. Int. Union Radio Sci. (URSI GASS), Aug. 2017, pp. 1-3.

[17] S. Hu, Y. Zhou, C. L. Law, and W. Dou, "Study of a Uniplanar Monopole Antenna for Passive Chipless UWB-RFID Localization System," IEEE Trans. Antennas Propag., vol. 58, no. 2, pp. 271-278, Feb. 2010.

[18] R. Anee and N. C. Karmakar, "Chipless RFID Tag Localization," IEEE Trans. Microw. Theory Techn., vol. 61, no. 11, pp. 4008-4017, Nov. 2013.

[19] R. Rezaiesarlak and M. Manteghi, "A Space-Frequency Technique for Chipless RFID Tag Localization," IEEE Trans. Antennas Propag., vol. 62, no. 11, pp. 5790-5797, Nov. 2014.
[20] L. Shahid et al., "Chipless RFID Tag for Touch Event Sensing and Localization," IEEE Access, vol. 8, pp. 502-513, 2020.

[21] S. Deif and M. Daneshmand, "Multiresonant Chipless RFID Array System for Coating Defect Detection and Corrosion Prediction," IEEE Trans. Ind. Electron., vol. 67, no. 10, pp. 8868-8877, Oct. 2020

[22] M. M. Khan, F. A. Tahir, M. F. Farooqui, A. Shamim, and H. M. Cheema, "3.56-bits/ $\mathrm{cm}^{2}$ Compact Inkjet Printed and Application Specific Chipless RFID Tag," IEEE Antennas Wireless Propag. Lett., vol. 15, pp. 1109-1112, 2016.

[23] C. E. Baum, E. J. Rothwell, K. M. Chen, and D. P. Nyquist, "The singularity expansion method and its application to target identification," Proc. IEEE, vol. 79, no. 10, pp. 1481-1492, Oct. 1991.

[24] T. K. Sarkar and O. Pereira, "Using the matrix pencil method to estimate the parameters of a sum of complex exponentials," IEEE Antennas Propag. Mag., vol. 37, no. 1, pp. 48-55, Feb. 1995.

[25] A. T. Blischak and M. Manteghi, "Embedded Singularity Chipless RFID Tags," IEEE Trans. Antennas Propag., vol. 59, no. 11, pp. 3961-3968, Nov. 2011.

[26] R. Rezaiesarlak and M. Manteghi, "Short-Time Matrix Pencil Method for Chipless RFID Detection Applications," IEEE Trans. Antennas Propag., vol. 61, no. 5, pp. 2801-2806, May 2013.

[27] R. Rezaiesarlak and M. Manteghi, "Complex-Natural-ResonanceBased Design of Chipless RFID Tag for High-Density Data," IEEE Trans. Antennas Propag., vol. 62, no. 2, pp. 898-904, Feb. 2014.

[28] R. Rezaiesarlak and M. Manteghi, "Accurate Extraction of Early/Late-Time Responses Using Short-Time Matrix Pencil Method for Transient Analysis of Scatterers," IEEE Trans. Antennas Propag., vol. 63, no. 11, pp. 4995-5002, Nov. 2015.

[29] F. Costa et al., "Robust Reading Approach for Moving Chipless RFID Tags by Using ISAR Processing," IEEE Trans. Microw. Theory Techn., vol. 66, no. 5, pp. 2442-2451, May 2018.

[30] G. Khadka, M. A. Bibile, L. M. Arjomandi, and N. C. Karmakar, "Analysis of Artifacts on Chipless RFID Backscatter Tag Signals for Real World Implementation," IEEE Access, vol. 7, pp. 66821-66831, 2019.

[31] F. Babaeian and N. C. Karmakar, "Time and Frequency Domains Analysis of Chipless RFID Back-Scattered Tag Reflection," IoT, vol. 1, no. 1, pp. 109-127, 2020.

[32] J. Kracek, M. Svanda, and K. Hoffmann, "Scalar Method for Reading of Chipless RFID Tags Based on Limited Ground Plane Backed Dipole Resonator Array," IEEE Trans. Microw. Theory Techn., vol. 67, no. 11, pp. 4547-4558, Nov. 2019.

[33] A. Ramos, E. Perret, O. Rance, S. Tedjini, A. Lázaro, and D. Girbau, "Temporal Separation Detection for Chipless Depolarizing Frequency-Coded RFID," IEEE Trans. Microw. Theory Techn., vol. 64, no. 7, pp. 2326-2337, Jul. 2016.

[34] A. Ramos, Z. Ali, A. Vena, M. Garbati, and E. Perret, "Single-Layer, Flexible, and Depolarizing Chipless RFID Tags," IEEE Access, vol. 8, pp. 72929-72941, 2020.

[35] J. Aliasgari and N. C. Karmakar, "Mathematical Model of Chipless RFID Tags for Detection Improvement," IEEE Trans. Microw. Theory Techn., vol. 68, no. 10, pp. 4103-4115, Oct. 2020.

[36] A. Vena, E. Perret, and S. Tedjni, "A Depolarizing Chipless RFID Tag for Robust Detection and Its FCC Compliant UWB Reading System," IEEE Trans. Microw. Theory Techn., vol. 61, no. 8, pp. 2982-2994, Aug. 2013.

[37] O. Rance, E. Perret, R. Siragusa, and P. Lemaitre-Auger, RCS Synthesis for Chipless RFID: Theory and Design. Elsevier, 2017.

[38] R. Rezaiesarlak and M. Manteghi, "Design of Chipless RFID Tags Based on Characteristic Mode Theory (CMT)," IEEE Trans. Antennas Propag., vol. 63, no. 2, pp. 711-718, Feb. 2015.

[39] J. Allen, "Applications of the short time Fourier transform to speech processing and spectral analysis," in Proc. 7th IEEE Int. Conf. Acoust., Speech, Signal Process., May 1982, pp. 1012-1015.

[40] K. Gröchenig, Foundations of Time-Frequency Analysis. Birkhäuser Boston, 2001.

[41] Z. Ali et al., "Detection of Minimum Geometrical Variation by FreeSpace-Based Chipless Approach and its Application to Authentication," IEEE Microw. Wireless Compon. Lett., vol. 28, no. 4, pp. 323-325, 2018.

[42] A. Vena, E. Perret, and S. Tedjini, Chipless RFID based on RF Encoding Particle: Realization, Coding and Reading System. Elsevier, 2016. 
[43] R. T. de Alencar, N. Barbot, M. Garbati, and E. Perret, "Characterization of Chipless RFID Tag in a 3-Dimensional Reading Zone," in Proc. IEEE Int. Symp. Antennas Propag. USNC-URSI Radio Sci. Meeting, Atlanta, GA, USA, 2019, pp. 639-640.

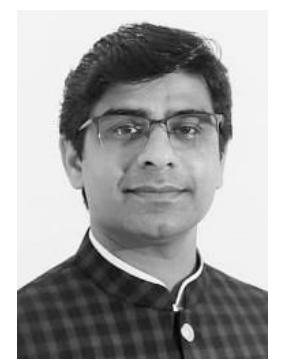

Zeshan Ali received the M.S. degree in electrical engineering from King Saud University (KSU), Riyadh, Saudi Arabia, and the Ph.D. degree in optical and radio frequency engineering from the Univ. Grenoble Alpes, Grenoble, France, in 2015 and 2019, respectively. From 2011 to 2016, he was a researcher with the Department of Electrical Engineering KSU. In 2016, he started his research at the Laboratoire de Conception et d'Intégration des Systèmes (LCIS), GrenobleINP - Institute of Engineering Univ. Grenoble Alpes, Valence, France and focused on the development of chipless RFID systems for product authentication applications. Since 2019, he has been a post-doctoral fellow with the LCIS, Valence, France. His current research interests include the design of RF/microwave circuits for chipless RFID tags and systems, radar signal processing, multi-band filters, reconfigurable filters and optimization methods. Zeshan Ali was a recipient of the Honorable Mention from the IEEE MTT France chapter for the student paper competition at the IEEE International Microwave Symposium (IMS) held in June 2018 at Philadelphia, PA, USA.

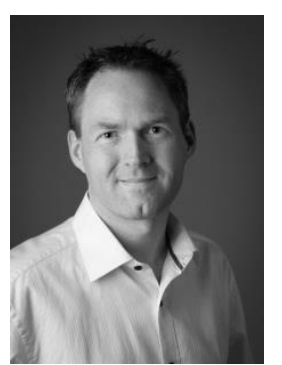

Etienne Perret (S'02-M'06-SM'13) received the Eng. Dipl. degree in electrical engineering from the Ecole Nationale Supérieure d'Electronique, d'Electrotechnique, d'Informatique, d'Hydraulique, et des Télécommunications, Toulouse, France, 2002, and the M.Sc. and Ph.D. degrees in electrical engineering from the Toulouse Institute of Technology, Toulouse, in 2002 and 2005, respectively. From 2005 to 2006, he held a post-doctoral position with the Institute of Fundamental Electronics, Orsay, France. In 2006, he was appointed Associate Professor of electrical engineering at Grenoble INP Institute of Engineering Univ. Grenoble Alpes, France. From 2014 to 2019, he has been a Junior Member with the Institut Universitaire de France, Paris, France, an institution that distinguishes professors for their research excellence, as evidenced by their international recognition. From 2015 to 2020, he has been an Appointed Member of the French National Council of Universities. He has authored or coauthored more than 200 technical conferences, letters and journal papers, and books and book chapters. He holds several patents. His works have generated more than 3000 citations. His current research interests include electromagnetic modeling of passive devices for millimeter and submillimeter-wave applications, and wireless communications, especially RFID and chipless RFID, and also include advanced computer-aided design techniques based on the development of an automated codesign synthesis computational approach. Dr. Perret is a Technical Program Committee member of the IEEE International Conference on RFID, the IEEE RFID TA; and a member of the IMS Technical Paper Review Committee. He was a recipient of several awards like the MIT Technology Review's French Innovator's under 35 in 2013, the French Innovative Techniques for the Environment Award in 2013, the SEE/IEEE Leon Brillouin Award for his outstanding achievement in the identification of an object in an unknown environment using a chipless label or tag in 2016, the IEEE MTT-S 2019 Outstanding Young Engineer Award and the Prix Espoir IMT - Académie des sciences in 2020. He was a Keynote Speaker and the Chairman of several international symposiums. Etienne Perret was awarded an ERC Consolidator Grant in 2017 for his project ScattererID.

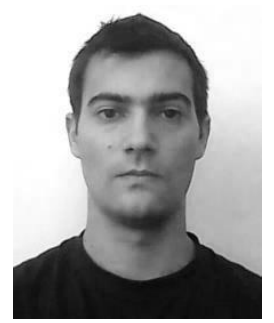

Nicolas Barbot received the M.S. degree and Ph.D. degree from University of Limoges, France. His Ph.D. work in Xlim laboratory was focused on error-correcting codes for the optical wireless channel.

A post-doctoral work was also realized in joint source-channel decoding at the L2S laboratory, at Gif sur Yvette, France.

Since September 2014, he has been an Assistant Professor at Grenoble INP - ESISAR at Valence, France. His research interest at LCIS laboratory includes backscattering communications, RFID, chipless RFID and antenna theory. More recently, he investigates the use of chipless tag as lowcost, batteryless and robust sensors.

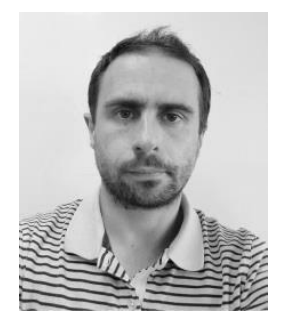

Romain Siragusa was born in Paris, France. $\mathrm{He}$ received the engineering and master's degrees from the Institute National Polytechnique de Grenoble, Grenoble Institute of Technology, Valence, France, in 2006, and the Ph.D. degree from the Laboratoire de Conception et d'Intégration des Systèmes, Grenoble Institute of Technology. From 2009 to 2010, he was a Post-Doctoral Fellow with the Commissariat à l'énergie atomique, Saclay, France, where he studied high-impedance surfaces for integrated antennas on silicon. Since 2011, he has been an Associate Professor with LCIS, Esisar, Grenoble Institute of Technology. His current research interests include leaky-wave structures and chipless RFID. 